\title{
MICROWAVE EMISSION OF SOLAR FLARES: CORONAL MASS EJECTIONS AND SHOCK WAVES
}

\author{
E.A.Isaeva ${ }^{1}$, Yu.T.Tsap ${ }^{2,3}$ \\ ${ }^{1}$ Institute of Radio astronomy of NAS of Ukraine, URAN-4 observatory, isaevaode@ gmail.com \\ ${ }^{2}$ Crimean Astrophysical Observatory \\ ${ }^{3}$ Pulkovo Observatory
}

ABSTRACT. Coronal mass ejections (CMEs) mostly affect the geomagnetic field. These structures are observed and studied with coronagraphic images therefore we don't see the corona in the plane of the sky and the measurements of the propagation speed for solar disk events are not accessible to coronagraphic observations. This suggests that microwave emission of solar flares that can be attributed to the gyrosynchrotron mechanism of mildly relativistic electrons can be used. In turn, the relationship between coronal shock waves and CMEs also remains unclear.

The data set that we use in this study is based on microwave $(\mu)$ observations of spectral fluxes $F_{\mu}$ at $8.8 \mathrm{GHz}$ obtained with the Radio Solar Telescope Network for the 124 proton solar events. The correlation coefficient $r$ between the CME velocities $V_{C M E}$ and integral fluxes of microwave emission $\int F_{\mu} d t$ achieves of about 0.8 while it does not exceed 0.36 between $V_{C M E}$ and the shock wave velocities. It has been found the quite strong correlation between the growth rate of microwave emission and the deceleration of frequency drift in the frequency range of $25-180 \mathrm{GHz}(r \approx 0.66)$. The obtained results suggest that microwave observation can be used to predict $V_{C M E}$ and the shock wave generation occurs in the region of flare energy release.

Keywords: Coronal mass ejections (CMEs), shock waves.

\section{Introduction}

The data set includes 124 proton events recorded from 06-11-1997 to 20-09-2015 with proton energies > 1-100 $\mathrm{MeV}$, which were simultaneously accompanied by the microwave IV type bursts ( $\mu$-bursts) and coronal mass ejections (CME).

This data set contains a large number of overlapping proton events, which were separated and identified by the authors themselves. In this connection, some of these events are absent in the world data center (WDC), where a list of proton events with proton energies $>10 \mathrm{MeV}$ is given and where overlapping events are not always separated. Among the 124 proton events 76 ones are present in the proton events directory ftp://ftp.swpc.noaa.gov/ pub/indices/SPE.txt, and 48 events have been identified by us independently. As a result, the list includes superimposed proton events with energy $\mathrm{E}>1-100 \mathrm{MeV}$.

When identifying proton events with flares, we were guided by generally accepted criteria. However, we do not exclude the mistakes made by us in the separation and identification of complex overlapping events.

The most important criterion for the proton flares is the presence of a $U$-shaped and $W$-shaped type of the frequency radio spectrum of continual radio bursts of IV type in the range of 25-25000 MHz. Such types of frequency radio spectrum have a deep minimum in the decimeter range and the pronounced maxima in the centimeter and meter ranges. Earlier it was shown by a number of authors that we can estimate the total number of accelerated particles based on the parameters of microwave bursts whereas the conditions of particle escape follow from the parameters of meter-decameter bursts (Akin'yan et al., 1977, 1978, Chertok 1982; Chertok et al., 1987; Melnikov et al., 1990; 1991; Lipatov et al., 2002). The sufficiently powerful meter-decameter component of the continuum radio bursts of the IV type suggests the favorable conditions (Daibog et al., 1987) and vice versa.

Another important criterion of for proton flares is the effective duration of microwave bursts at the frequency of a microwave spectral maximum. Using this parameter, we can estimate the enrichment of the solar energetic particles (SEP) by protons with respect to electrons. In particular, Daibog et al. $(1989,1993)$ have shown that the acceleration of sub-relativistic protons requires significantly more time than electrons.

The next important criterion of proton flares is the heliographic longitude of the proton flare $\lambda$, counted to the east and west of the central meridian on the Sun. It is known that there is a very strong dependence of the parameters of proton fluxes on the heliographic longitude of flares (Ochelkov, 1986), since the direction of propagation of the proton flux is determined by the spiral structure of the interplanetary magnetic field.

Thus, to identify proton events with flares, it is necessary to know the heliographic longitude of the flare and the type of the frequency radio spectrum.

It is known that the microwave IV type bursts ( $\mu$ bursts) are always accompanied by bursts of soft X-ray emission. Therefore, based on the time of the flare maximum and coordinates in X-rays, it is possible to identify the radio bursts on the solar disk with proton events.

Using the coordinates of the X-ray flare, we can estimate the arrival time of the first protons at the near-Earth orbit. In this case, it is necessary to take into account the time delay between the first protons detected near-Earth orbit and the peak of the X-ray flare emission. The corresponding delay time largely depends on the heliographic longitude of the flare. The shortest delay time is observed 
for western proton events with heliographic longitude in the range from 60 to 90 degrees, measured from the central meridian on the Sun. High-energy protons with E > $100 \mathrm{MeV}$ are registered the first in about 20 minutes after the peak of X-ray flare emission. However, the delay time for the eastern events is much longer than the delay time for the western ones and can range from a few hours to three days. The delay time, or the geometry of propagation of the proton flux in the interplanetary space, is determined by the degree of diffuse scattering of the proton flux. The longer proton path is caused by the more diffuse scattering, and as a consequence, the decrease in the intensity of the proton flux in the Earth's orbit. It should be taken into account that the fluxes of high-energy protons undergo the greatest attenuation. As a result that, the energy spectrum of the protons becomes steeper, that is, for eastern events, an increase in the exponent of the energy spectrum of the protons is observed. Moreover, the higher the degree of diffuse scattering of the proton flux, the greater the rise time of the proton flux, which in turn affects the temporal profiles of the proton flux. Therefore, for eastern events, the temporal profile of the intensity of the proton flux becomes smooth, and for the eastern events on the limb of the Sun, the growth time can be even longer than the decay time. At the same time, for western proton events a sharp increase in the intensity of the proton flux is observed for several hours.

The identification of proton events with flares, for single western proton events, is not very difficult. However, for a series of overlapping proton events, the identification procedure can be ambiguous, because in a series of overlapping events, both western and eastern events can be present, spread over time from several hours to 3 days. But, despite this, the vast majority of overlapping proton events can be divided and identified with flares.

To identify proton events with flares, we used the original data of X-ray emission from the Sun and the proton fluxes using GOES data, as well as the original records of the radio emission of the Sun in the range 25-15400 MHz.

\section{Background data}

The original data of the intensity of the proton flux with energy $>1-100 \mathrm{MeV}$ and the X-ray emission in the ranges $0.05-0.4$ and $0.1-0.8 \mathrm{~nm}$ (https://satdat.ngdc.noaa. gov/sem/goes/data/new_avg/).

Dynamic spectra in the range 25-180 $\mathrm{MHz}$ (http://www.ngdc.noaa.gov/stp/space-weather/solar-data/ solar-features/solar-radio/rstn-spectral/).

Solar radio emission at frequencies of 245, 410, 610, 1415, 2695, 4995, 8800, $15400 \mathrm{MHz}$ (https://www. ngdc.noaa.gov/stp/space-weather/solar-data/solar-features/ solar-radio/rstn-1-second/ ).

Tabular data of the shock wave velocities detected in the range 25-180 MHz (http://www.ngdc.noaa.gov/stp/ space-weather/solar-data/solar-features/solar-radio/radiobursts/tables/spectral-sgd/).

Tabular data of velocities of coronal mass ejections (CME) (https://cdaw.gsfc.nasa.gov/CME_list/UNIVERSAL/text ver/univ_all.txt).

\section{Relationship between CME velocities and para-} meters of continual radio bursts

In this paper, the relationship between the CME velocities $V_{C M E}$ and the parameters of continual radio bursts in the range $245-15400 \mathrm{MHz}$ is investigated. Comparative analysis has shown that there is a strong correlation between $V_{C M E}$ and the integral flux of microwave bursts $\int F_{\mu} d t$ in the range of 2695-15400 MHz. Fig. 1a shows the relationship between $V_{C M E}$ and $\int F_{\mu} d t$ at $8800 \mathrm{MHz}$, where $N$ is the number of events, and $r$ is the correlation coefficient between these values. For the vast majority of proton events, indicated by black circles, the correlation coefficient $r$ between $V_{C M E}$ and $\int F_{\mu} d t$ is about 0.80 . The gray line in Fig. 1a shows the linear regression (1), which is described by the equation

$$
\log _{10} V_{C M E, \mu}=0.2322 \cdot \log _{10} \int F_{\mu} d t+2.1108
$$

where $V_{C M E}, \mu$ is the calculated value. The light circles in Fig. 1a are most likely related to errors in the identification of proton events with flares and CMEs. Among 14 events marked with light circles, 7 ones were identified by the authors, the rest were taken from the WDC catalog. But, out of these 7 events, 3 in the microwave range 4995$15400 \mathrm{MHz}$ were accompanied by bursts of GRF type, but they had a sufficiently powerful continual burst in the range 245-2695 $\mathrm{MHz}$. In connection with this, these events were included in the sample of proton events. If 7 events identified by the authors and 3 events that were

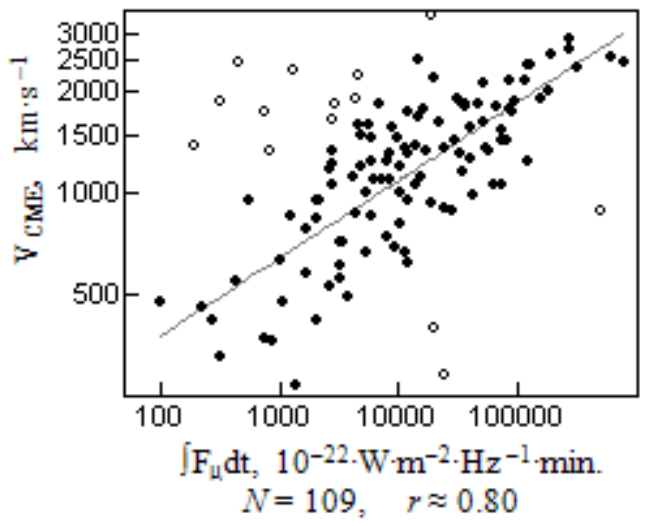

a).

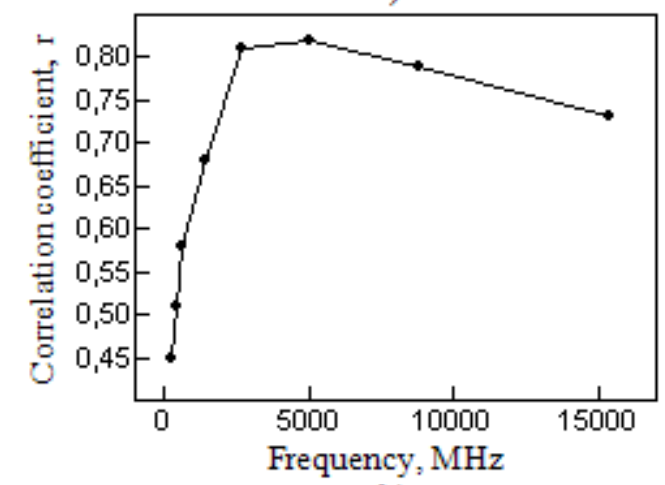

b).

Figure 1: The relationship between the CME velocities $V_{C M E}$ and the integral fluxes of continual radio bursts $\int F d t$. 
accompanied by GRF bursts, to exclude from this sample, then only 4 events will remain for which there is a poor correlation between $V_{C M E}$ and the integral flux of $\mu$ bursts.

It is also seen that the correlation between the $\mathrm{V}_{\mathrm{CME}}$ and the integral flux $\int F d t$ decreases significantly with decreasing frequency, and the correlation coefficient $r$ between $V_{C M E}$ and $\int F d t$ does not exceed 0.45 in the meter range at $245 \mathrm{MHz}$, (see Figure 1b).

\section{Relationship between coronal shock waves and CMEs}

To study the correlatiobetween shock waves and CMEs, the tabular data of the shock wave and CME velocities, as well as original dynamic spectra in the range 25-180 MHz, were used.

It is known that shock waves can be generated both by solar flares and coronal mass ejections. The II type bursts in the decimeter-meter ranges are associated with shock waves caused by flares (Wagner et al., 1983, Vrsnak et al., 1995), while the decameter-hectometre bursts is related to the propagation of interplanetary shock waves generated by CMEs (Gopalswamy et al., 1998; Classen et al., 2002). The most reliable indicator of shock waves in the solar

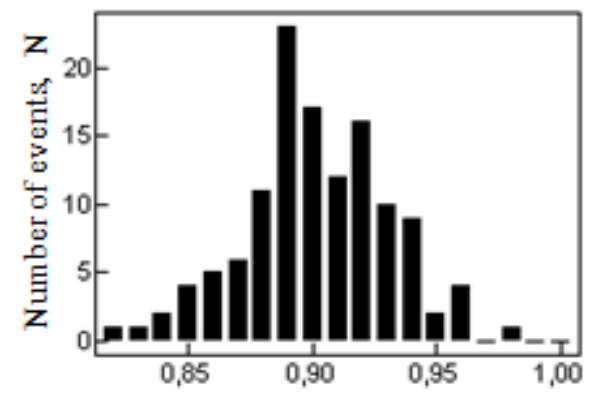

a). Correlation coefficient, $r$

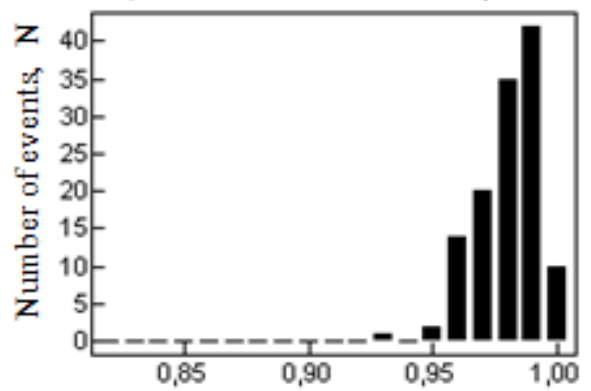

b). Correlation coefficient, $r$

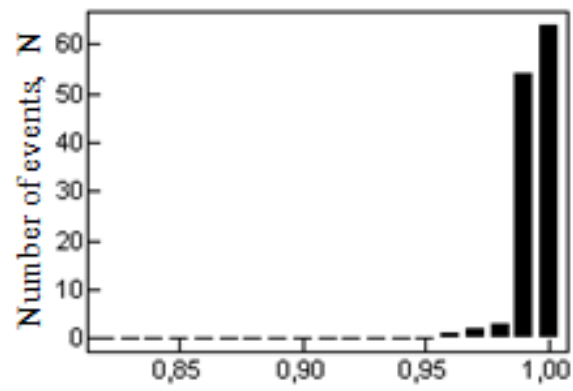

c). Correlation coefficient, $r$

Figure 2: The comparison of the different approximations describing the dependence of the frequency $f_{i, j}$ on the time $t_{i}$ corona are the slowly drifting type II bursts. It is believed, that the plasma mechanism of radio emission is responsible for their generation (Cairns et al., 2003).

In this paper, the following approximation of the dependence of the frequency $f_{i, j}$ on the time $t_{i}$ is used

$$
\log _{10} f_{i, j}=a_{j} \cdot \sqrt{t_{i}}+b_{j}
$$

where $t_{i}$ is the time of the maximum intensity of the II type burst at the frequency $f_{i, j}, a_{j}$ and $b_{j}$ are the linear regression coefficients, $i=1 \ldots n$ is the account number, $j=$ 1,2 is the harmonic number. The zero point of time for all events corresponds to the beginning of the first harmonic at a frequency of $180 \mathrm{MHz}$. The approximation (2) allows us to estimate the frequency $f_{i, j}$ in the range $25-180 \mathrm{MHz}$ for $95 \%$ of the events quite precisely. In particular, the correlation coefficient $r$ between the calculated $f_{i, j}$ and observed frequency values $f$ turned out to be greater than $>0.98$ for selected events (see Figure 2c). Fig. 2 shows the histograms of the distribution of the number of events for which the correlation coefficient $r$ between the observed value of $f$ and the calculated value of the frequency $f_{i, j}$ lies in a specified range of values. Other approximations $(3,4)$

$$
\begin{aligned}
& \log _{10} f_{i, j}=a_{j} \cdot \log _{10} t_{i}+b_{j} \\
& \log _{10} f_{i, j}=a_{j} \cdot t_{i}+b_{j}
\end{aligned}
$$

of the frequency dependence on time was previously used, which gave a lower accuracy of frequency estimation (see

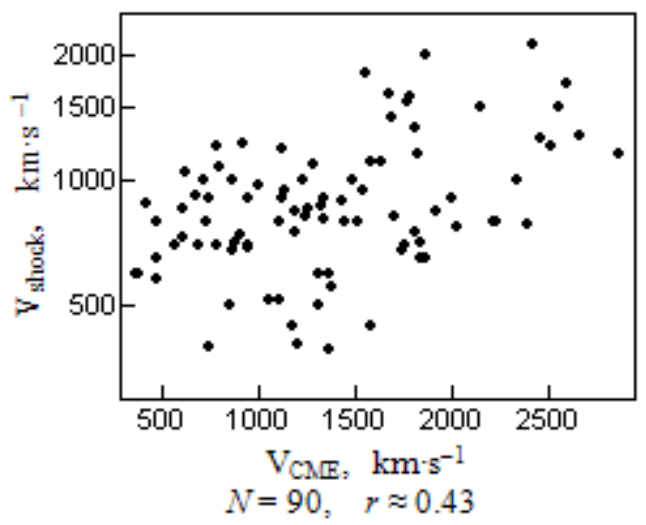

a).

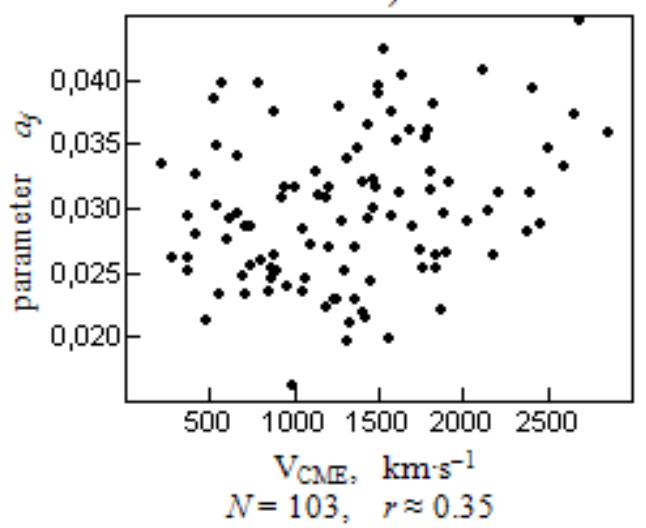

b).

Figure 3: The relationship between shock wave velocities $V_{\text {shock }}$ and CME velocities $V_{C M E}$. 
Figure 2a,b). In Fig. 3a,b shows the relationship between the CME velocities $V_{C M E}$, shock wave velocities $V_{\text {shock}}$, and the parameters $a_{j}$, which characterize the slowdown of frequency drift in the model (2). It is clear that the corresponding correlations are weak. At first glance, this can explain by different time of registration of shock wave velocities and CME. However, the correlation between the CME velocities $V_{C M E}$ and the frequency drift velocities $V_{I I}=\frac{d f}{d t}$, calculated at the time of CME detection turned out to be weak.

\section{Relationship between shock waves and solar flares}

Let us consider the correlation between shock waves and the parameters of $\mu$-bursts in terms of the original radio data at 8 fixed frequencies in the range 245-15400 MHz. A comparative analysis has shown that there is a relatively good correlation between the parameters $a_{j}$ and the rise time $t_{\mu}$ of $\mu$-bursts at the frequency of $8800 \mathrm{MHz}$. The correlation coefficient between $t_{\mu}$ and $a_{j}$ is $\approx 0.66$ (see Figure 4a). Note that we took into account that flares differ significantly in intensity and duration we therefore expressed the rise time $t_{\mu}$ as a percentage of the duration (100\%) of $\mu$-bursts.

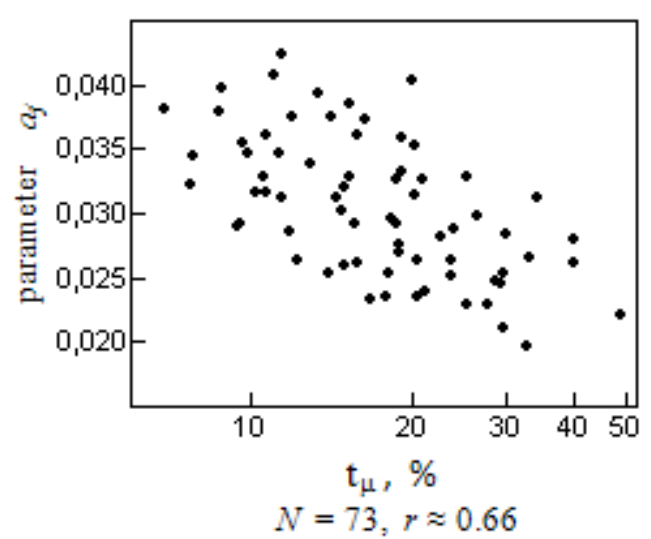

a).

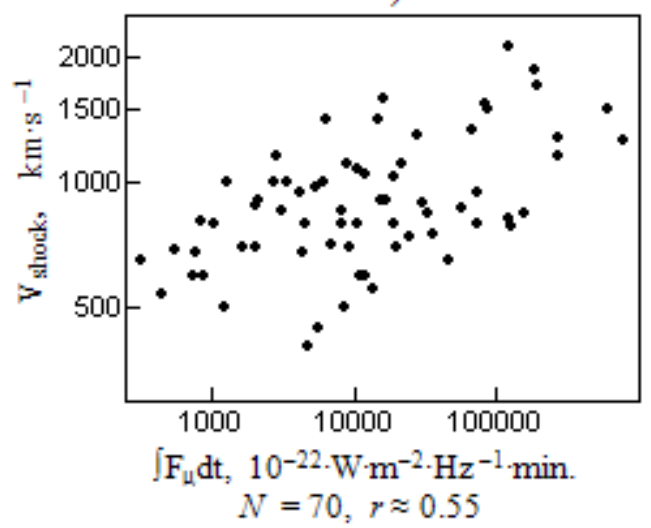

b).

Figure 4: Relationship between shock wave velocities $V_{\text {shock }}$ and parameters of microwave bursts
The correlation between the shock wave velocities $V_{\text {shock }}$ and the integral fluxes $\int F_{\mu} d t$ of $\mu$-bursts at the frequency of $8800 \mathrm{MHz}$ was also investigated. The correlation coefficient between $\int F_{\mu} d t$ and $V_{\text {shock }} \approx 0.55$ (see Fig. 4b). The weak correlation between $V_{\text {shock }}$ and $\int F_{\mu} d t$ is most likely due to the fact that different stations give averaged estimates of the velocity of shock waves at different time a fairly large dispersion should be observed. Despite this, the correlation between $\int F_{\mu} d t$ and $V_{\text {shock }}$ can by quite clearly seen for the majority of proton events (see Fig. 4b).

\section{References}

Akin'yan S.T. et al.: 1977, Geomagnetizm i Aeronomiia, 17, 10.

Akin'yan S.T. et al.: 1978, Geomagnetizm i Aeronomiia, 18, 577.

Cairns I.H. at al.: 2003, Space Sci. Rev., 107, 27.

Chertok I.M.: 1982, Geomagnetizm i Aeronomiia, 22, 182.

Chertok I.M. et al.: 1987, Geomagnetizm i Aeronomiia, 27, 362.

Classen H.T. et al.: 2002, $A \& A, \mathbf{3 8 4}, 1098$.

Daibog E.I. et al.: 1987, Izv. AN SSSR, ser.ph., 51, 1825.

Daibog E.I. et al.: 1988, Izv. AN SSSR, ser.ph., 52, 2403.

Daibog E.I. et al.: 1989, Letters to the Astron. J., 15, 991.

Daibog E.I. et al.: 1993, Solar Phys., 144, 361.

Gopalswamy et. al.: 1998, J. Geophys. Res., 103, 307.

Lipatov B.N. et al.: 2002, Izv. of HEIs, Radiophysics, 65 , № 2,83 .

Melnikov V.F. et al.: 1990, Solar-Terrestrial Predictions, US Dep. Of Comm., NOAA, Boulder, Co., 1, 533.

Melnikov V.F., et al.: 1991, Space Research., 29, 95.

Ochelkov Yu.P.: 1986, Geomagnetism and Aeronomy, 26, № 6, 1007 .

Vrsnak B. et al.: 1995, Solar Phys., 158, 331.

Wagner W.J. et al.: 1983, $A \& A, \mathbf{1 2 0}, 136$. 